Article

\title{
Design and Fabrication of a Novel MEMS Relay with Low Actuation Voltage
}

\author{
Hao $\mathrm{Li}^{1}{ }^{1}$, , Yong Ruan ${ }^{1, *}$, Zheng You ${ }^{1}$ and Zhiqiang Song ${ }^{2}$ \\ 1 State Key Laboratory of Precision Measurement Technology and Instruments, Department of Precision \\ Instrument, Tsinghua University, Haidian District, Beijing 100084, China; lihaothu@tsinghua.edu.cn (H.L.); \\ yz-dpi@mail.tsinghua.edu.cn (Z.Y.) \\ 2 MEMS Institute of Zibo National High-Tech Industrial Development Zone, Zibo 255000, \\ China; songzqmic@163.com \\ * Correspondence: ruanyong@mail.tsinghua.edu.cn; Tel.: +86-10-62776000
}

Received: 9 December 2019; Accepted: 30 January 2020; Published: 7 February 2020

\begin{abstract}
Compared with conventional solid-state relays, micro-electro mechanical system (MEMS) relays have the advantages of high isolation, low contact resistance, low power consumption, and abrupt switching characteristics. Nevertheless, the widespread application of MEMS relays has been limited due to the issue of the conflict between low actuation voltages and high device performance. This article presents a novel cantilever MEMS relay with an embedded contact electrode which helps to achieve a low actuation voltage (below $8 \mathrm{~V}$ ) and high restoring force simultaneously. Meanwhile, the contact resistance is as low as around $0.4 \Omega$ and the reliability is verified. To thoroughly investigate and analyze the novel cantilever MEMS relay, a static theoretical model of the structure was developed. Based on the model, the cantilever MEMS relay was designed and optimized. Then, the relays were fabricated by the bulk-silicon micromachining process based on the silicon-glass anodic bonding technology. Finally, the switching performance of the novel cantilever MEMS relay was measured. Experimental results demonstrate that the proposed MEMS relay has a low actuation voltage below $8 \mathrm{~V}$ and high performance, which is in good agreement with the simulation results, and shows significant advantages when compared with previous reports. Therefore, the proposed MEMS relay with an embedded contact electrode is promising in practical applications.
\end{abstract}

Keywords: micro-electro mechanical system (MEMS) relay; cantilever; low actuation voltage

\section{Introduction}

Micro-electro mechanical system (MEMS) relays have the potential to be applied in space technology, communication, and automatic devices [1]. Compared to conventional solid-state relays, MEMS relays have the advantages of high isolation, low on-resistance, low power consumption, and abrupt switching characteristics [2]. Besides, they are reliable and inexpensive to facilitate packaging and system integration as they can be batch manufactured like solid-state relays [3,4].

Among various driving methods for MEMS relays, electrostatic actuation is mostly utilized owing to the advantages of low power and easy fabrication [5]. Nevertheless, electrostatic MEMS relays have the issue of the conflict between low actuation voltages and good device performance. In general, the low actuation voltage implies a low contact force or low restoring force. The former leads to a high contact resistance [6], while the latter contributes to an easily irreversible stiction [7]. Different ideas have been proposed to reduce the actuation voltage without lowing the contact force or restoring force. For example, novel spring structures have been exploited to reduce the actuation voltage [8,9], but the structural and fabrication complexity is increased. Another method to lower the driving voltage requires an additional pre-charged electrode $[10,11]$. 
Component reliability is another issue for electrostatic MEMS relays. While stiction is a major cause for low reliability, numerous studies have dedicated efforts to enhance restoring force to overcome stiction. Oberhammer has designed a novel mechanism to acquire a large active opening force, but the contact force is decreased [12]. Increasing the electrode area is another mechanical approach to enhance restoring force that does not sacrifice the contact force or actuation voltage. However, it requires a bigger size [13]. Other researches utilize special active anti-stiction mechanisms to provide extra restoring force $[7,14,15]$, but they increase the structural and fabrication complexity.

In this article, we present a novel cantilever MEMS relay with an embedded contact electrode. This embedded contact electrode structure helps to achieve a low actuation voltage (below $8 \mathrm{~V}$ ). Meanwhile, the contact resistance is as low as around $0.4 \Omega$ and the switching-on time and switching-off time are lower than $100 \mu \mathrm{S}$. To thoroughly investigate and analyze the novel cantilever MEMS relay, a static theoretical model of the structure was developed. Based on the model, the cantilever MEMS relay was designed and optimized. Then, the relays were fabricated by the bulk-silicon micromachining process based on the silicon-glass anodic bonding technology. Finally, the switching performance of the novel cantilever MEMS relay was measured. The experimental results demonstrate that the MEMS relay has a low actuation voltage and high performance.

\section{Design of the Cantilever Micro-Electro Mechanical System (MEMS) Relay}

Figure 1 illustrates the proposed cantilever relay with an embedded contact electrode and a conventional cantilever switch. The designed MEMS relay is comprised of a hollow suspended spring, a driving plate, and a contact electrode. The hollow suspended spring lowers the actuation voltage and enhances the device stability considerably, which has been demonstrated in our earlier work [4].

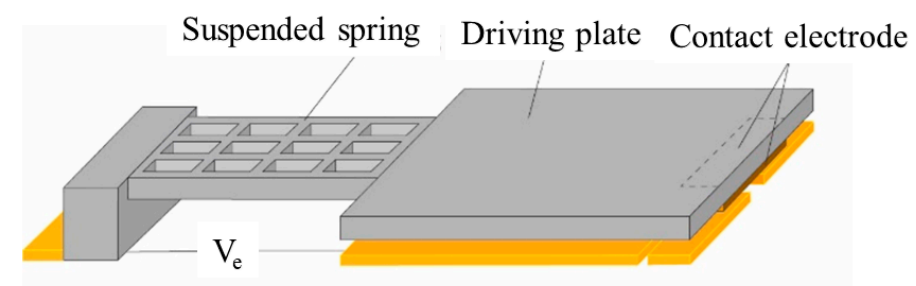

(a)

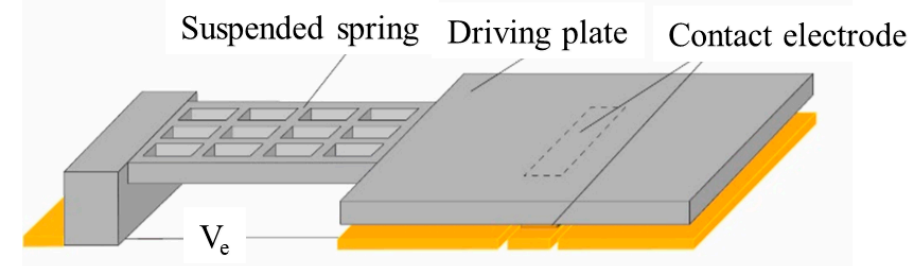

(b)

Figure 1. Schematic structure of the proposed micro-electro mechanical system (MEMS) relay for an individual element: (a) Contact electrode at the edge of the cantilever relay; (b) cantilever relay with an embedded contact electrode. 


\section{Modeling and Simulation}

\subsection{Static Modeling}

With the low stiffness of the suspended spring and high stiffness of the driving plate, the driving plate can be regarded as a rigid body. As shown in Figure $2 d$, Point 1 is the joint between the spring and the plate, $\omega_{1}$ is its deflection, and $\theta_{1}$ is its rotation angle. The deflection at Point $x$ can be derived:

$$
y=\omega_{1}+\theta_{1} x_{1}
$$

where $x_{1}$ designates the location of Point $x$ as shown in Figure $2 \mathrm{~d}$. Therefore, the magnitude of the electrostatic force at Point $x$ can be determined (Point $x$ is not at the contact electrode):

$$
q_{x}=\varepsilon_{0} \frac{V_{e}^{2}}{2\left(g_{0}-y\right)^{2}} W_{2}
$$

where $\varepsilon_{0}$ is the permittivity of air, $g_{0}$ is the original air gap between the cantilever beam and the gate electrode, $V_{\mathrm{e}}$ is the driving voltage, and $W_{2}$ is the plate width. $M_{q x}$ can be derived as:

$$
M_{q_{x}}=\varepsilon_{0} \frac{V_{e}^{2}}{2\left(g_{0}-y\right)^{2}} W_{2} \cdot x_{1}
$$

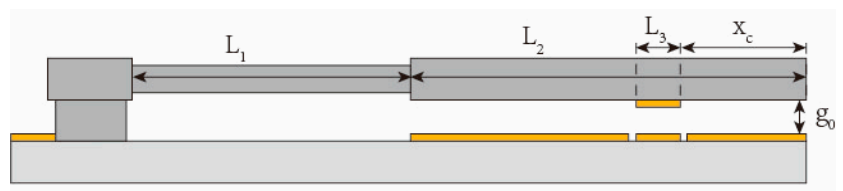

(a)

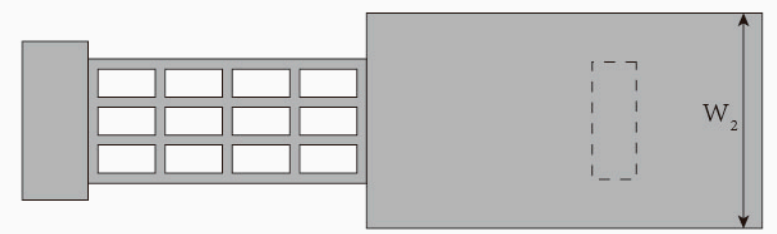

(b)

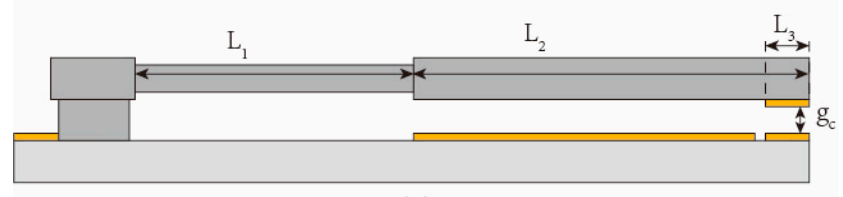

(c)

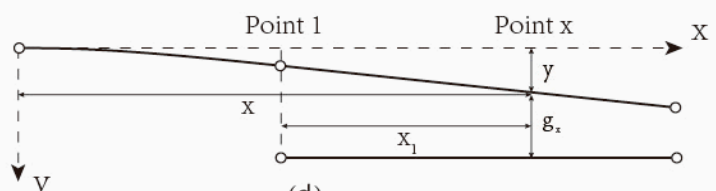

(d)

Figure 2. The equivalent parameters of the proposed MEMS relay: $(\mathbf{a}-\mathbf{c})$ Dimension marking of geometric parameters for the cantilever; (d) establishment of coordinate for geometric parameters.

The electrostatic forces along the plate are equivalent to a concentrated load at Point 1, which can be determined by integrating Equations (2) and (3):

$$
F_{e}=\int_{0}^{L_{2}} \varepsilon_{0} \frac{V_{e}^{2}}{2\left(g_{0}-y\right)^{2}} W_{2} d x_{1}-\int_{L_{2}-L_{3}-x_{c}}^{L_{2}-x_{c}} \varepsilon_{0} \frac{V_{e}^{2}}{2\left(g_{0}-y\right)^{2}} W_{2} d x_{1}
$$




$$
M_{e}=\int_{0}^{L_{2}} \varepsilon_{0} \frac{V_{e}^{2}}{2\left(g_{0}-y\right)^{2}} W_{2} x_{1} d x_{1}-\int_{L_{2}-L_{3}-x_{c}}^{L_{2}-x_{c}} \varepsilon_{0} \frac{V_{e}^{2}}{2\left(g_{0}-y\right)^{2}} W_{2} x_{1} d x_{1},
$$

where $x_{c}$ designates the location of the contact electrode as shown in Figure 2a. The deflection and rotation angle at Point 1 can be derived:

$$
\begin{aligned}
& \omega_{1}=\frac{F_{e} L_{1}^{3}}{3 E I}+\frac{M_{e} L_{1}^{2}}{2 E I}, \\
& \theta_{1}=\frac{F_{e} L_{1}^{2}}{2 E I}+\frac{M_{e} L_{1}}{E I},
\end{aligned}
$$

where $E$ and $I$ are the Young's modulus and the second moment of inertia of the suspended spring, and $L_{1}$ is the spring length. When the driving voltage Ve is small, the deflection of the relay can be determined by numerical calculation by Equations (1)-(7). If there is no solution for Equations (1)-(7), $V_{\mathrm{e}}$ reaches the pull-in voltage.

The finite element model was established by COMSOL (version 5.4, COMSOL Co., Ltd., Stockholm, Sweden). The structural parameters of the traditional and proposed MEMS relay are listed in Table 1. We change the position of contact electrode and keep the other parameters unchanged to verify the effect of promoting the relay performance.

Table 1. Geometric parameters of the proposed MEMS relay.

\begin{tabular}{ccc}
\hline Symbol & Description of Parameters & Value \\
\hline$L_{1}$ & $\begin{array}{c}\text { Length of the hollow spring } \\
\text { Equivalent Width of the hollow } \\
\text { spring }\end{array}$ & $460 \mu \mathrm{m}$ \\
$W_{1}$ & $\begin{array}{c}\text { (coupled with four } 20 \mu \mathrm{m} \text { wide } \\
\text { microbeams) }\end{array}$ & $80 \mu \mathrm{m}$ \\
$L_{2}$ & Length of the driving plate & $540 \mu \mathrm{m}$ \\
$W_{2}$ & Width of the driving plate & $330 \mu \mathrm{m}$ \\
$L_{3}$ & Length of the contact electrode & $50 \mu \mathrm{m}$ \\
$W_{3}$ & Width of the contact electrode & $180 \mu \mathrm{m}$ \\
$t$ & Thickness of the cantilever beam & $22 \mu \mathrm{m}$ \\
$g_{0}$ & Distance of the air gap & $1.5 \mu \mathrm{m}$ \\
$g_{\mathrm{c}}$ & Distance of the contact gap & $0.7 \mu \mathrm{m}$ \\
$\mathrm{x}_{\mathrm{c}}$ & Position of the contact electrode & $0-250 \mu \mathrm{m}$ \\
\hline
\end{tabular}

Figure 3 illustrates the simulated results of the actuation voltage. The simulation results show that the actuation voltage decreases with the contact electrode moving inside or becoming smaller. According to Figure 3, when the length of the contact electrode is $50 \mu \mathrm{m}$ as designed, the actuation voltage drops from $8.02 \mathrm{~V}$ to $7.81 \mathrm{~V}$ when the contact electrode of the conventional relay moves $250 \mu \mathrm{m}$ inside. The decrement is bigger if the area of the contact electrode becomes bigger. 


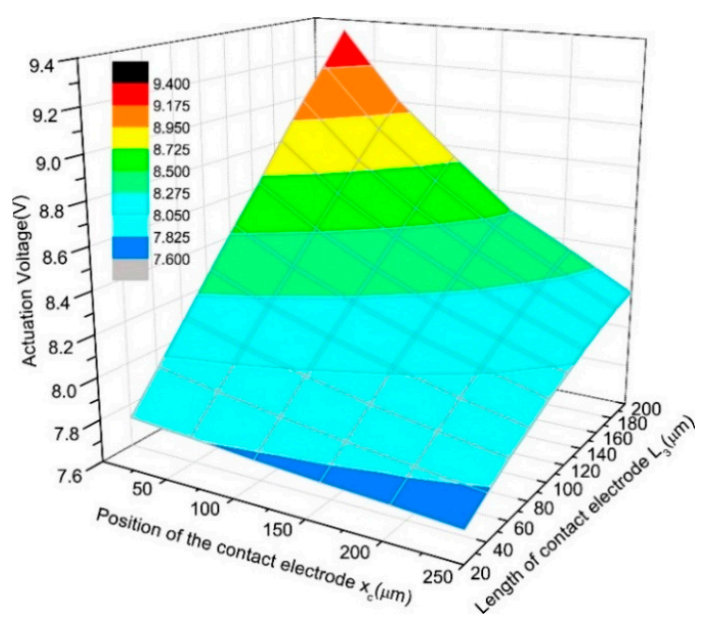

Figure 3. Simulated actuation voltage versus $x_{c}$ and $L_{3}$.

We also simulated the contact force and restoring force against the value of $x_{c}$. We imposed the same actuation voltage of $9 \mathrm{~V}$, and Figure 4 shows that the contact force increases dramatically with $x_{c}$ increasing. Then, we solved the minimal force imposed on the contact electrode that ensured the relay close. The minimal force, which is regarded as the restoring force when the relay keeps closed, also increases with $x_{\mathcal{c}}$ increasing.

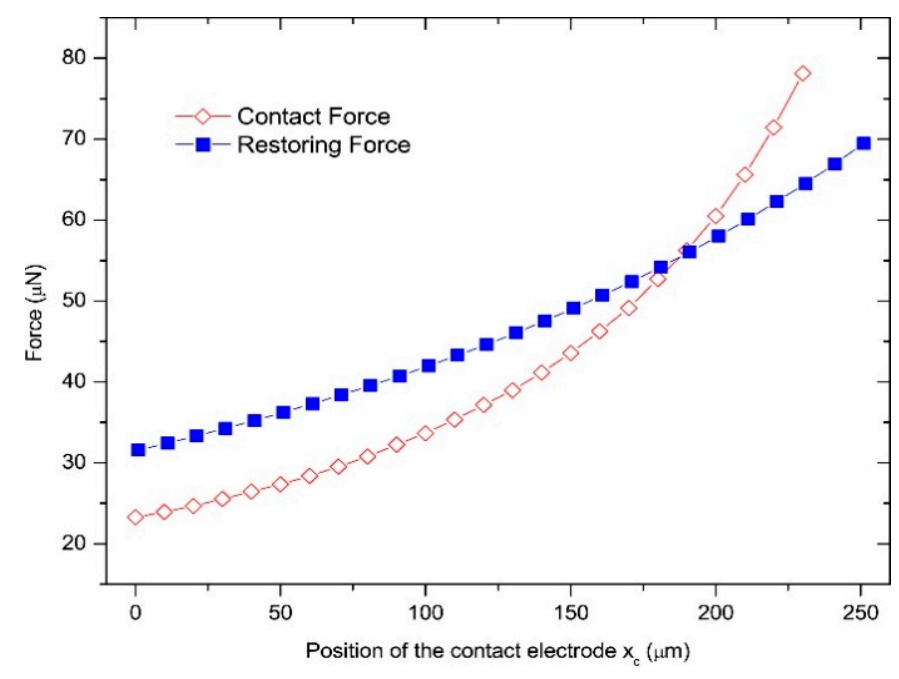

Figure 4. Simulated contact force and restoring force against $x_{c}$.

\subsection{Dynamic Modeling}

The dynamic model is established using a similar way which has been introduced in earlier work. The dynamic Euler-Bernoulli beam equation can describe the transient response of the cantilever relay:

$$
m \frac{\partial^{2} y}{\partial t^{2}}+D \frac{\partial y}{\partial t}+E I \frac{\partial^{4} y}{\partial x^{4}}=F_{e}-F_{c}
$$

where $m$ is the mass per unit length of the cantilever relay, $y(x, t)$ is the downward deflection of the relay at time $t, D$ is the damping factor, $E I$ is the flexural rigidity, $F_{e}$ is the electrostatic force, and $F_{c}$ is the contact force. The initial and boundary conditions are as follows:

$$
\left\{\begin{array}{l}
y(x, 0)=0 \\
\frac{\partial y(x, 0)}{\partial t}=0
\end{array}\right.
$$




$$
\left\{\begin{array}{l}
y(0, t)=0, \frac{\partial y(0, t)}{\partial x}=0, \\
\frac{\partial^{2} y(l, t)}{\partial x^{2}}=0 .
\end{array}\right.
$$

Since the suspended spring of the relay is hollowed, the air damping of the spring can be ignored. It is assumed that the relay operates in an air medium, then the air damping of the driving plate can be simplified as:

$$
D=K W_{2} \frac{\mu}{1+9.638\left(\lambda_{0} / g_{0}\right)^{1.159}} \frac{L_{2}{ }^{2}}{\left(g_{0}-y\right)^{3}} .
$$

where $K$ is the flow coefficient, which is 0.013 in our design; $\mu$ is the air damping coefficient, and $\lambda_{0}$ is the mean free path of the air molecules $(\approx 64 \mathrm{~nm})$.

The electrostatic force is:

$$
F_{e}=\varepsilon_{0} \frac{V_{e}^{2}}{2\left(g_{0}-y\right)^{2}} W_{2} .
$$

The contact force can be approximated by a linear spring model:

$$
F_{c}=k_{c}\left(y-g_{c}\right) \cdot H\left(y-g_{c}\right), x \in\left[L_{1}+L_{2}-L_{3}-x_{c}, L_{1}+L_{2}-x_{c}\right] \text {, }
$$

where the Heaviside function $H\left(y-g_{c}\right)$ ensures that the force is only applied when the relay makes contact. The spring constant kc takes an empirical value, ensuring the contact deformation is small.

The dynamic model was simulated by COMSOL. Figure 5 illustrates the simulated dynamic responses of the conventional and proposed MEMS relay of different contact electrode positions. Being imposed the same driving voltage of $15 \mathrm{~V}$, the dynamic bounce and switching time are both suppressed when the contact electrode is moving inside. Particularly, the switching time of the conventional relay $\left(x_{c}=0 \mu \mathrm{m}\right)$ is about 2.8 time units, while the time is diminished to 1.8 time units when $x_{c}$ is $200 \mu \mathrm{m}$ as Figure 5 shows. When the contact electrode is moving inside, it is surrounded by the driving plate. Thus, the electrostatic force around the contact electrode restrains the contact bounces, which further reduces the switching time. As is shown in Figure 5, the optimal $x_{c}$ is about $100 \mu \mathrm{m}$, which has the minimum contact bounce time.

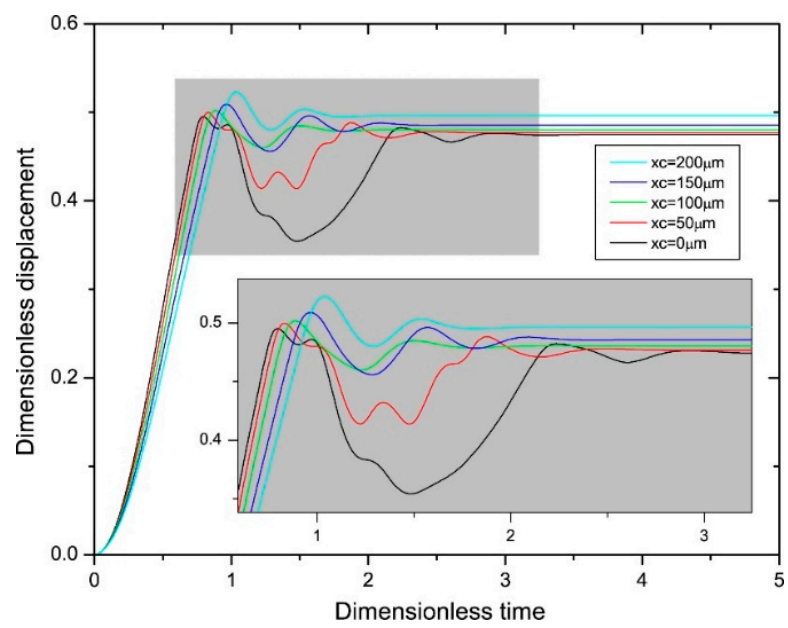

Figure 5. The simulated dynamic responses of the conventional and proposed MEMS relay of different contact electrode positions.

\section{Fabrication for MEMS Relay}

The proposed cantilever relays with embedded contact electrodes were fabricated using bulk-silicon techniques based on the silicon-glass anodic bonding to form and pattern the mechanical and actuation structures. The fabrication process is summarized in Figure 6. First, a silicon wafer with a polished surface was patterned and etched to a depth of $1.5 \mu \mathrm{m}$. Next, another step of $1.0 \mu \mathrm{m}$ was 
etched to form the dimple for the contact electrode. Then, a $4000 \AA \mathrm{SiO}_{2}$ insulating layer was deposited on the silicon device layer, and then the unexposed region of the $\mathrm{SiO} 2$ layer was etched to a depth of $2000 \AA$. Next, a Cr $(400 \AA) / \mathrm{Au}(10,700 \AA) / \mathrm{Pt}(200 \AA) / \mathrm{Au}(1000 \AA) / \mathrm{Pt}(200 \AA) / \mathrm{Au}(500 \AA)$ metal layer $(13,000 \AA$ in total) was sputtered and patterned by a lift-off process. Then, the $\mathrm{SiO} 2$ layer was etched to a depth of $2000 \AA$ to remove the unexposed region. On the other side, a Pyrex 7740 glass wafer was etched 12,500 A step. Then, the same metal layer as in Step (d) was sputtered to form the electrodes and leads. Next, the silicon layer after Step (e) was anodically bonded to the glass substrate after Step (g) and thinned to $22 \mu \mathrm{m}$. Finally, the device layer was etched by ICP (Inductively Coupled Plasma) to release the relay structures.
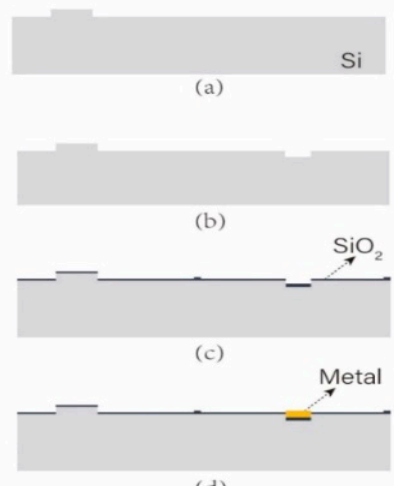

(d)

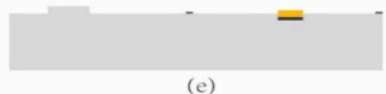

(e)

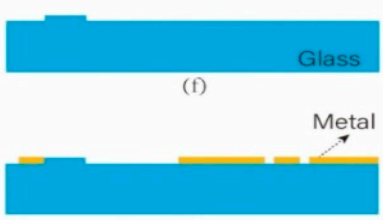

$(\mathrm{g})$

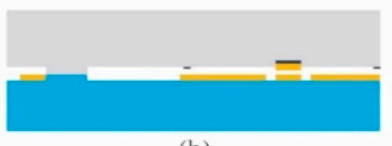

(h)

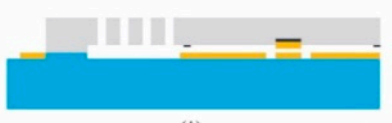

(i)

(a)

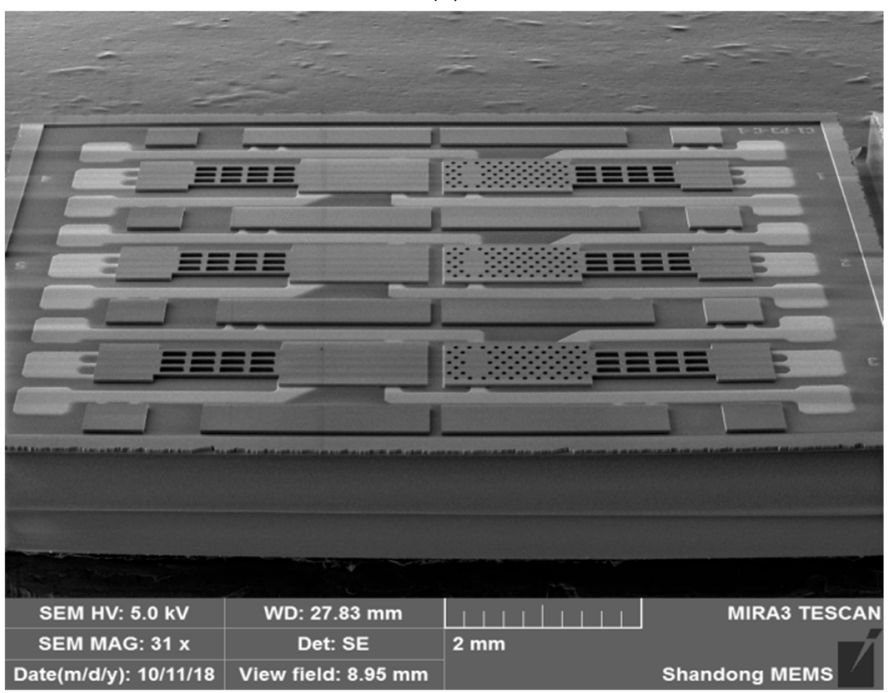

(b)

Figure 6. Microfabrication process and SEM image of the MEMS relay array. (a) Microfabrication process of the proposed MEMS relay array; (b) SEM image of the MEMS relay array.

\section{Experimental Results and Discussion}

\subsection{Test Experiment Platform}

Figure 7 shows the experimental platform for electrical performance of the proposed MEMS relay. It contains a semiconductor analyzer (4200-SCS, Tektronix Inc., Johnston, OH, USA), a manual probe station (M8, Semiprobe Inc., Winooski, VT, USA), a precision power supply (B2902A, 
Agilent Technologies Inc., Santa Clara, CA, USA), and a digital oscilloscope (Agilent Technologies Inc., Santa Clara, CA, USA). The drive and load terminals of the proposed relay are connected to the manual probe station. The semiconductor analyzer applies a scanning voltage of 0 to $15 \mathrm{~V}$ to the drive terminals and records the hysteresis loop of pull-in and pull-off voltage. The digital oscilloscope is used to record the switching-on time, switching-off time, and switching state when the pull-in voltage and pull-off voltage are attained. A precision power supply and semiconductor analyzer are used to measure the contact resistance.

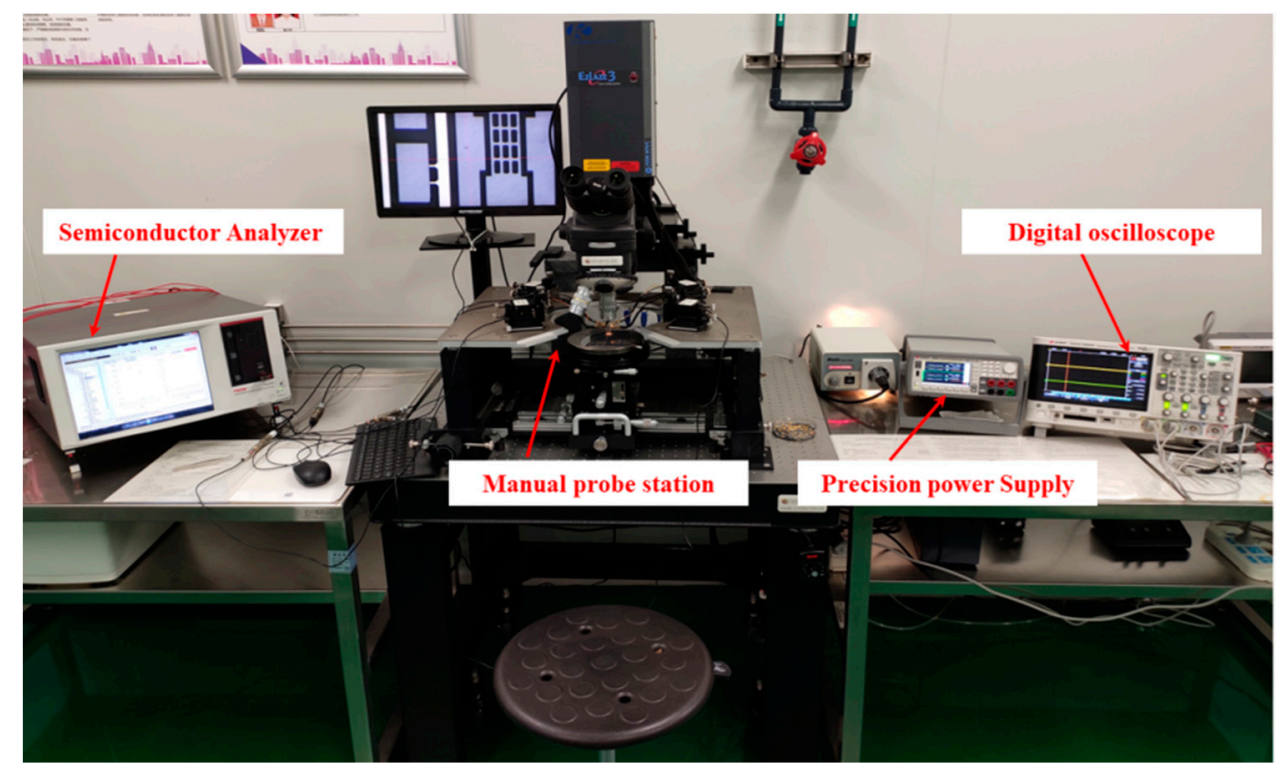

Figure 7. Experiment platform for electrical performance.

\subsection{Pull-In and Pull-Off Voltage}

The proposed MEMS relay uses voltage as the excitation quantity, and the actuation voltage refers to the corresponding voltage value that makes the MEMS relay act. According to the characteristics of MEMS relay, it includes the minimum action voltage, which is also called pull-in voltage, and the maximum release voltage, which is called pull-off voltage. Figure 8 shows the setup schematic for measuring the actuation voltage.

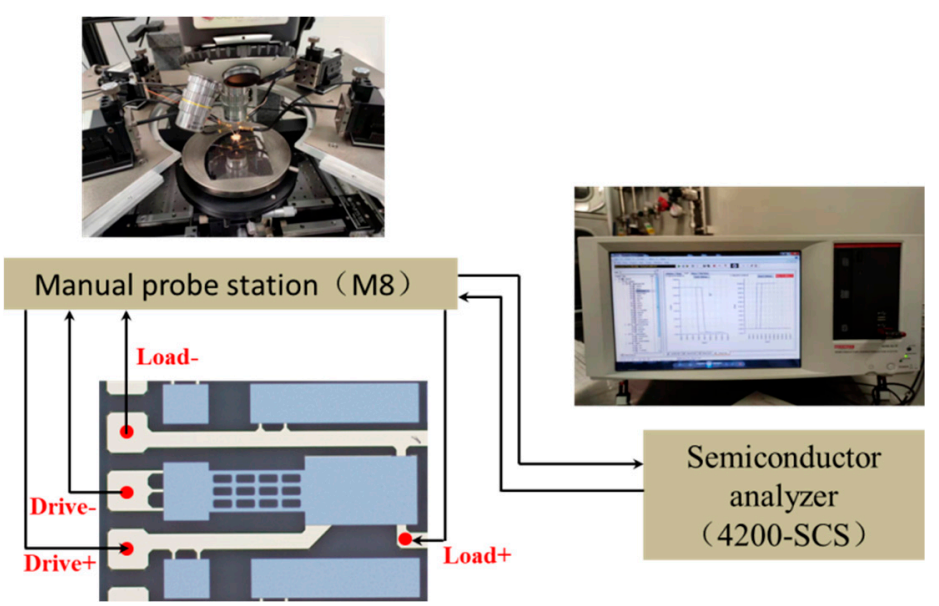

Figure 8. The setup schematic for measuring the actuation voltage. 
Experimental results show that the average pull-in voltages are about 7.5-8.0 V, which is very similar to the simulated voltages of 7.81-8.02 V. The variation among relays with different contact electrode positions are not distinguishable due to the fabrication error. This is comprehensible because the fabrication error is of just $0.1 \mu \mathrm{m}$, which has a significant effect on the pull-in voltage with the air gap being designed as $1.5 \mu \mathrm{m}$. However, the actuation voltage of $8 \mathrm{~V}$ is low enough for applicability. The average pull-off voltages are about 5.5-6.0 V, which are lower than the pull-in voltages. The actuation process of a relay is shown in Figure 9. In this figure, the measured pull-in voltage and pull-off voltages are $7.5 \mathrm{~V}$ and $6 \mathrm{~V}$ respectively.

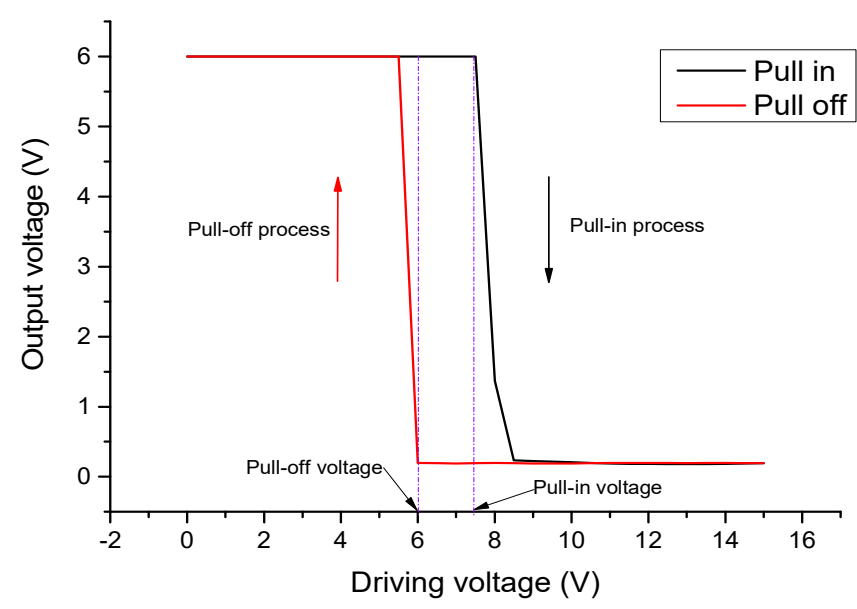

Figure 9. Hysteresis loop of pull-in and pull-off voltage.

\subsection{Switching-On and Switching-Off Time}

Switching-on time refers to the duration from the time when the pull-in voltage of the MEMS relay attained to the time when the state of the proposed relay changed to be ON. Switching-off time refers to the duration from the time when the pull-off voltage of the MEMS relay attained in the pull-off process to the time when the state of the proposed relay changed to be OFF. Figure 10 shows the setup schematic for measuring the switching-on and switching-off time. The driving power supply applies a scanning voltage of 0 to $10 \mathrm{~V}$ to the drive terminals and the load power supply voltage set to be $5 \mathrm{~V}$. During the experiment, the digital oscilloscope records the waveform change of voltages.

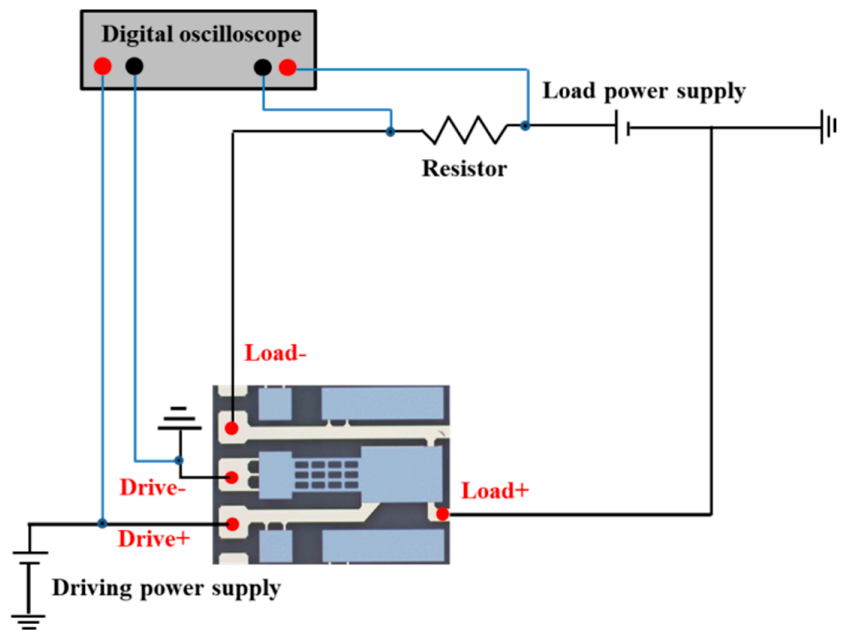

Figure 10. The setup schematic for measuring the switching-on and switching-off time.

Figure 11 shows the result of the switching-on time measurement experiment. As shown in Figure 11, the measured switching-on time of the proposed relay is about $75 \mu \mathrm{S}$. During the pull-in 
process, when the actuation voltage attains to $7.5 \mathrm{~V}$ (pull-in voltage), the driving plate of cantilever begins to bend to the contact electrode. After $75 \mu \mathrm{S}$, the circuit gets connected, and the voltage at both ends of the resistor reaches $5 \mathrm{~V}$.

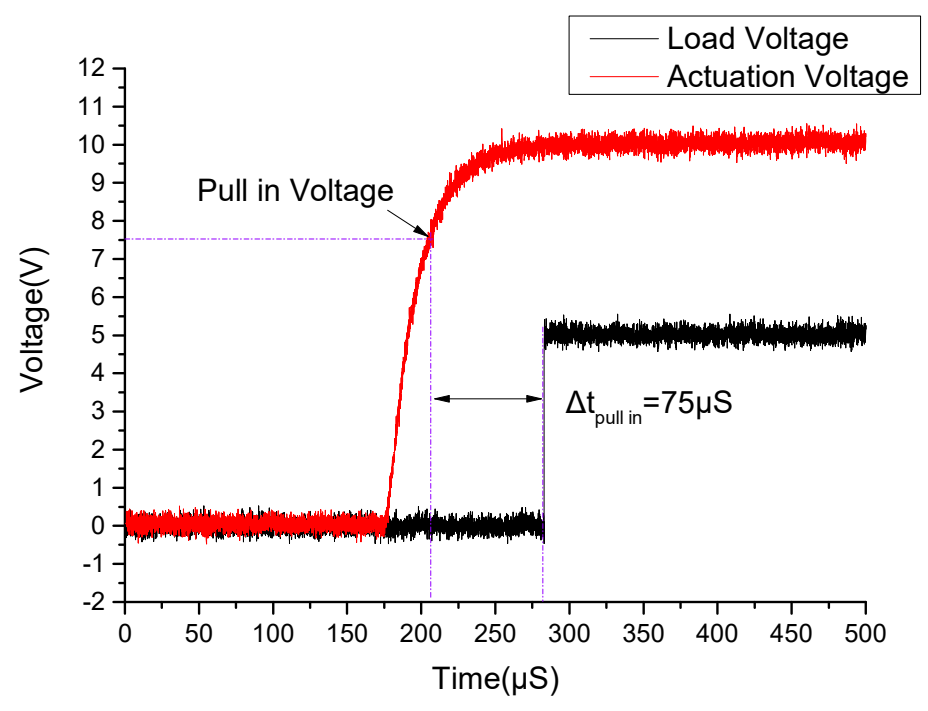

Figure 11. The measured switching-on time of the proposed MEMS relay.

Figure 12 shows the result of the switching-off time measurement experiment. As is shown in Figure 12, the measured switching-off time of the proposed relay is about $25 \mu \mathrm{S}$. During the pull-off process, when the actuation voltage drops to $6 \mathrm{~V}$ (pull-off voltage), the driving plate of cantilever begins to rebound to balance position. After $25 \mu \mathrm{S}$, the circuit gets disconnected, and the voltage at both ends of the resistor turns to $0 \mathrm{~V}$.

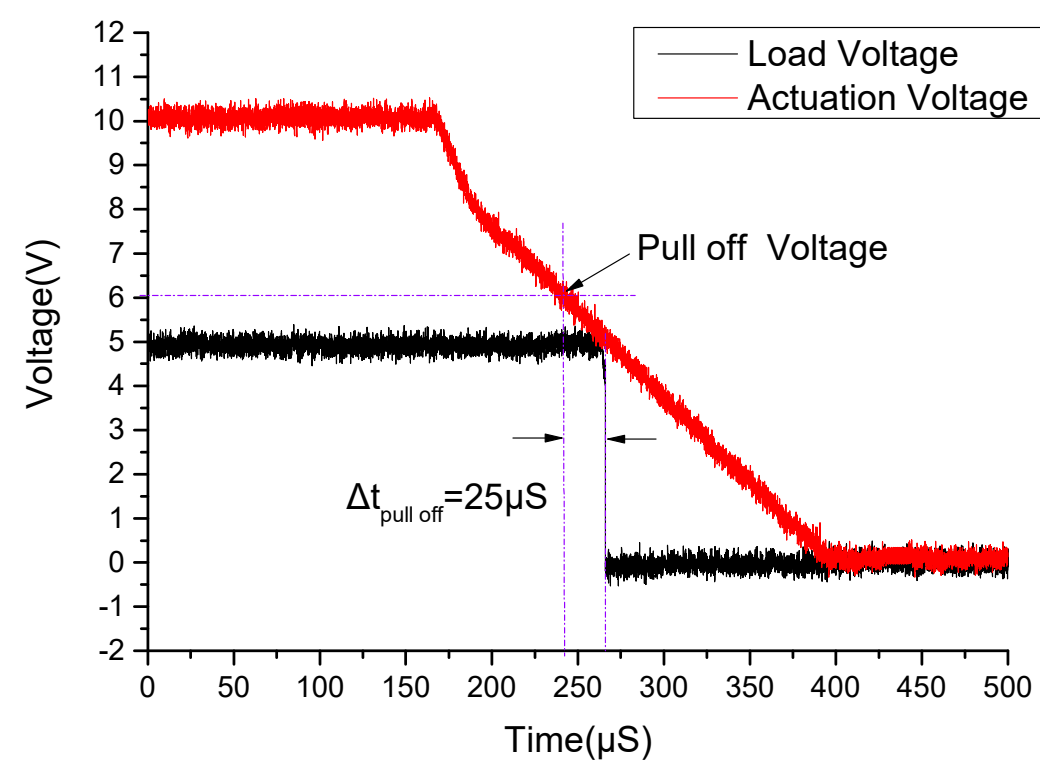

Figure 12. The measured switching-off time of the proposed MEMS relay.

\subsection{Contact Resistance}

In this research, the contact resistance of the proposed relay is measured using the Kelvin four-wire method. For each test point, there is a constant current source and a voltage detection unit, which are strictly separated, and constitute an independent loop. The voltage line must be connected to a test loop with extremely high input impedance. Meanwhile, the current flowing through the detection 
line is extremely small, which is approximately zero. The constant current source (B2902A) provides a constant current. The current passes through the contact electrode. Figure 13 shows the setup schematic for measuring the contact resistance. In this figure, the driving power supply provides a constant $10 \mathrm{~V}$ voltage to make sure the MEMS relay keeps on. The precision power supply provides a constant current. The digital oscilloscope measures the voltage across the relay. The contact resistance can be expressed as $R=V / I$.

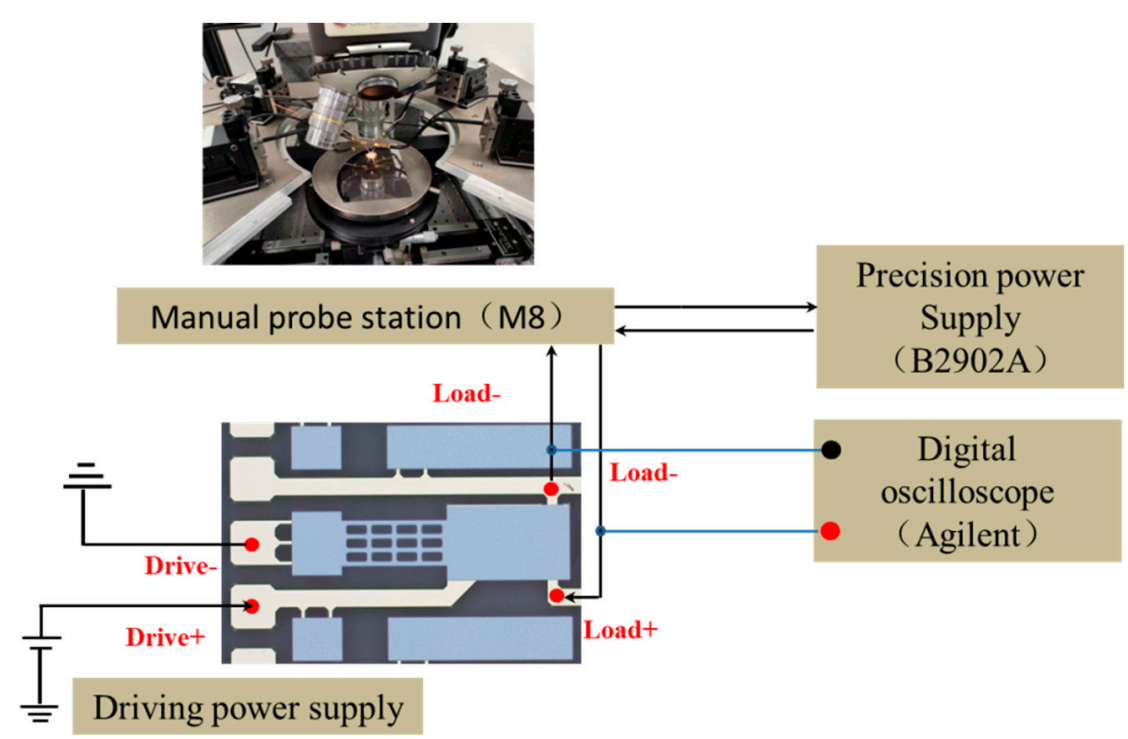

Figure 13. The setup schematic for measuring the contact resistance.

In order to reduce measurement error, the measurements of the contact resistance were carried out and averaged. Stable and low contact resistance can be obtained when the pull-in voltage is $8 \mathrm{~V}$. The measured contact resistance of each relay was less than $0.4 \Omega$. The contact resistance was also measured under different load currents. The experimental result shows that the contact resistance was lower at a load current of $20 \mathrm{~mA}$ than that at $200 \mu \mathrm{A}$. This was due to the softening of the contact asperities, which may result in a more effective contact area; however, it increased slightly at currents higher than $20 \mathrm{~mA}$, which may be caused by the resistivity increasing induced by the high temperature at localized asperities.

\subsection{Contact Lifetime}

The contact lifetime was measured on the manual probe station by the semiconductor analyzer (4200-SCS). The source/measure unit provides two independent channels: one channel was used for providing the driving voltage, the other for measuring the load circuit resistance. The driving voltage was set at $15 \mathrm{~V}$ which has a low contact resistance and rapid response. MEMS relays are mostly operated in two modes: cold-switching operation and hot-switching operation. Cold-switching refers to relay closure before applying voltage and voltage removal before relay opening, while hot-switching refers to the relay actuation synchronized with the electrical switching. Figure 14 shows the setup schematic for measuring the contact lifetime. 


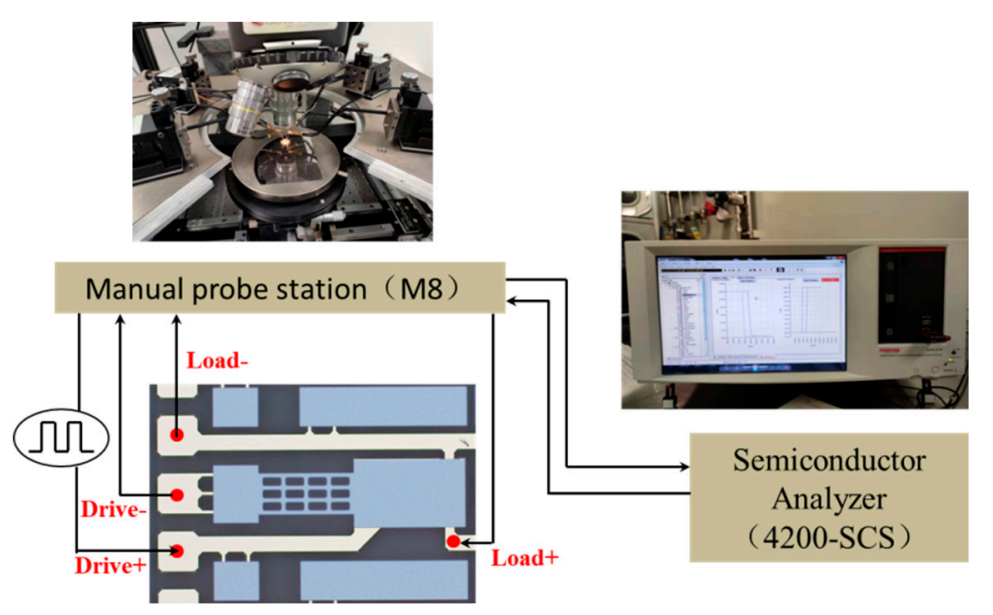

Figure 14. The setup schematic for measuring the contact lifetime.

In the cold-switching operation experiment, the load of the semiconductor analyzer was set at $30 \mathrm{mV}$ and $30 \mu \mathrm{A}$. After a $5 \times 10^{6}$ test cycle, the contact resistance of the MEMS relay rises to above $10 \Omega$, which means a failure of the relay. This may be due to the formation of an insulating film on the contact surface. On the other hand, when the load was set at $12 \mathrm{~V}$ and $20 \mathrm{~mA}$, the test was carried out about 6000 cycles before the MEMS relay reached failure.

\subsection{Comparison of the Performance}

Table 2 makes a comparison of the performance for MEMS relays reported in the literature and this research. From this table, we can conclude that the proposed MEMS relay in this research achieves a lower actuation voltage (below $8 \mathrm{~V}$ ) than the MEMS relays reported in the literature; meanwhile, the contact resistance, switching time, and contact lifetime performance show a certain degree of advantage.

Table 2. Comparison of the performance for MEMS relays reported in literature.

\begin{tabular}{ccccc}
\hline Research Institute & $\begin{array}{c}\text { Actuation Voltage } \\
(\mathbf{V})\end{array}$ & $\begin{array}{c}\text { Contact } \\
\text { Resistance }(\boldsymbol{\Omega})\end{array}$ & $\begin{array}{c}\text { Switching Time } \\
(\boldsymbol{\mu} \mathbf{S})\end{array}$ & Contact Lifetime \\
\hline MIT [16] & 20 & 0.05 & $20,000-50,000$ & - \\
KAIST [17] & 40 & 0.005 & 230 & $4.9 \times 10^{5}$ \\
UCSD [18] & $75-90$ & 1.5 & $<10$ & - \\
ADI [19] & 80 & 1.6 & $<30$ & $10 \times 10^{9}$ \\
This research & $<8$ & 0.4 & $<75$ & $5 \times 10^{6}$ \\
\hline
\end{tabular}

\section{Conclusions}

In this article, we present a novel cantilever MEMS relay with an embedded contact electrode. This embedded contact electrode structure helps to achieve a low actuation voltage (below $8 \mathrm{~V}$ ) and high device performance simultaneously. To thoroughly investigate and analyze the novel cantilever MEMS relay, a static theoretical model of the structure was developed. Based on the model, the cantilever MEMS relay was designed and optimized. Then, the relays were fabricated by the bulk-silicon micromachining process based on the silicon-glass anodic bonding technology. Finally, the switching performance of the novel cantilever MEMS relay was measured. The experimental results demonstrate that the MEMS relay has a low actuation voltage and high performance.

Author Contributions: Conceptualization, Y.R. and Z.Y.; data curation, Z.S.; formal analysis, H.L.; funding acquisition, Y.R.; investigation, H.L.; methodology, Y.R.; project administration, Y.R.; resources, Z.S.; software, H.L.; supervision, Y.R.; visualization, Y.R.; writing — original draft preparation, H.L.; writing—review and editing, H.L. All authors have read and agreed to the published version of the manuscript. 
Funding: This research was funded by the Key Research and Development Program of Shandong Province (grant number: 2017GGX30138) and Zibo City Innovation and Development Key Project (grant number: 2016CX19A024).

Conflicts of Interest: The authors declare no conflict of interest.

\section{References}

1. Rebeiz, G.M.; Patel, C.D.; Han, S.K.; Ko, C.H.; Ho, K.M. The search for a reliable MEMS switch. IEEE Microw. Mag. 2013, 14, 57-67. [CrossRef]

2. Kim, M.W.; Song, Y.H.; Yoon, J.B. Modeling, fabrication and demonstration of a rib-type cantilever switch with an extended gate electrode. J. Micromech. Microeng. 2011, 21, 115009. [CrossRef]

3. Lee, H.S.; Leung, C.H.; Shi, J.; Chang, S.C.; Lorincz, S.; Nedelescu, L. Integrated microrelays: Concept and initial results. J. Microelectromech. Syst. 2002, 11, 147-153.

4. Ma, B.; You, Z.; Ruan, Y.; Chang, S.; Zhang, G. Electrostatically actuated MEMS relay arrays for high-power applications. Microsyst. Technol. 2016, 22, 911-920. [CrossRef]

5. Gao, Y.; You, Z.; Zhao, J. Electrostatic comb-drive actuator for MEMS relays/switches with double-tilt comb fingers and tilted parallelogram beams. J. Micromech. Microeng. 2015, 25, 045003. [CrossRef]

6. Kim, M.W.; Song, Y.H.; Ko, S.D.; Ahn, S.J.; Yoon, J.B. Ultra-low voltage MEMS switch using a folded hinge structure. Micro Nano Syst. Lett. 2014, 2, 1-5. [CrossRef]

7. Kim, M.W.; Song, Y.H.; Yang, H.H.; Yoon, J.B. An ultra-low voltage MEMS switch using stiction-recovery actuation. J. Micromech. Microeng. 2013, 23, 045022. [CrossRef]

8. Pacheco, S.P.; Katehi, L.P.; Nguyen, C.C. Design of low actuation voltage RF MEMS switch. In Proceedings of the 2000 IEEE MTT-S International Microwave Symposium Digest, Boston, MA, USA, 11-16 June 2000; pp. 165-168.

9. Balaraman, D.; Bhattacharya, S.K.; Ayazi, F.; Papapolymerou, J. Low-cost low actuation voltage copper RF MEMS switches. In Proceedings of the 2002 IEEE MTT-S International Microwave Symposium Digest, Seattle, WA, USA, 2-7 June 2002; pp. 1225-1228.

10. Yang, H.H.; Lee, J.O.; Yoon, J.B. Maneuvering pull-in voltage of an electrostatic micro-switch by introducing a pre-charged electrode. In Proceedings of the 2007 IEEE International Electron Devices Meeting, Washington, DC, USA, 10-12 December 2007; pp. 439-442.

11. Amponsah, K.; Yoshimizu, N.; Ardanuc, S.; Lal, A. Near-kT switching-energy lateral NEMS switch. In Proceedings of the 2010 IEEE 5th International Conference on Nano/Micro Engineered and Molecular Systems, Xiamen, China, 20-23 January 2010; pp. 985-988.

12. Oberhammer, J.; Stemme, G. Active opening force and passive contact force electrostatic switches for soft metal contact materials. J. Microelectromech. Syst. 2006, 15, 1235-1242. [CrossRef]

13. Mercado, L.; Kuo, S.M.; Lee, T.Y.T.; Liu, L. A mechanical approach to overcome RF MEMS switch stiction problem. In Proceedings of the 53rd Electronic Components A Technology Conference, New Orleans, LA, USA, 27-30 May 2003; pp. 377-384.

14. Touati, S.; Lorphelin, N.; Kanciurzewski, A.; Robin, R.; Rollier, A.S.; Millet, O.; Segueni, K. Low actuation voltage totally free flexible RF MEMS switch with antistiction system. In Proceedings of the 2008 Symposium on Design, Test, Integration and Packaging of MEMS/MOEMS, Nice, France, 9-11 April 2008; pp. 66-70.

15. Iannacci, J.; Repchankova, A.; Faes, A.; Tazzoli, A.; Meneghesso, G.; Dalla Betta, G.F. Enhancement of RF-MEMS switch reliability through an active anti-stiction heat-based mechanism. Microelectron. Reliab. 2010, 50, 1599-1603. [CrossRef]

16. Wong, J.E. Analysis, Design, Fabrication, and Testing of a MEMS Switch for Power Applications. Ph.D. Thesis, Massachusetts Institute of Technology, Cambridge, MA, USA, 2000.

17. Song, Y.H.; Han, C.H.; Kim, M.W.; Lee, J.O.; Yoon, J.B. An electrostatically actuated stacked-electrode MEMS relay with a levering and torsional spring for power applications. J. Microelectromech. Syst. 2012, 21, 1209-1217. [CrossRef] 
18. Patel, C.D.; Rebeiz, G.M. An RF-MEMS Switch with mN Contact Forces. In Proceedings of the 2010 IEEE MTT-S International Microwave Symposium, Anaheim, CA, USA, 23-28 May 2010; pp. 1242-1245.

19. ADGM1304 Datasheet and Product Info. Available online: https://www.analog.com/cn/products/adgm1304. html (accessed on 25 January 2020).

(C) 2020 by the authors. Licensee MDPI, Basel, Switzerland. This article is an open access article distributed under the terms and conditions of the Creative Commons Attribution (CC BY) license (http://creativecommons.org/licenses/by/4.0/). 